\title{
A New Anthraquinone Glycoside from Rhamnus nakaharai and Anti-tyrosinase Effect
}

\section{of 6-Methoxysorigenin}

Tzy-Ming Lu ${ }^{1, *}$ and Horng-Huey Ko ${ }^{2}$

${ }^{1}$ Department of Pharmacy, Tajen University, Pingtung 907, Taiwan $;{ }^{2}$ Department of Fragrance and Cosmetic Science, Kaohsiung Medical University, Kaohsiung 807, Taiwan

* Corresponding author: Department of Pharmacy, Tajen University; No. 20 Wei-Xin Rd., Yanpu, Pingtung 90741, Taiwan; Tel: 886-8-7624001 ext. 2727; Fax: 886-8-7625308; e-mail: tzminglu@gmail.com; or $\underline{\text { cmlu@mail.tajen.edu.tw }}$ 


\section{A New Anthraquinone Glycoside from Rhamnus nakaharai and Anti-tyrosinase Effect of 6-Methoxysorigenin}

In continual study on the heartwood of Rhamnus nakaharai, a new alaternin-8-O-glucoside, namely 1,2,6,8-tetrahydroxy-3-methylanthraquinone-8-O- $\beta$-glucopyranoside (1), together with some known compounds were further isolated and characterized by $1 \mathrm{D}, 2 \mathrm{D}$ NMR and other spectral evidences. The free radical scavenging and anti-tyrosinase activities of the isolates including alaternin (1a), emodin (2a), emodin-8-O- $\beta$-glucopyranoside (2), 6-methoxysorigenin-8-O- $\beta$-glucopyranoside (3), and 6-methoxysorigenin (3a) were tested. Alaternin (1a) exhibited to be mild DPPH radical scavenger with half as potent as vitamin C, while both alaternin (1a) and emodin-8-O- $\beta$-glucopyranoside (2) exhibited stronger SOD-like activity than that of BHA. 6-Methoxysorigenin (3a), a reported potential antioxidant, and its 8-O-glucoside (3) both performed significant inhibitory effect on mushroom tyrosinase with about twice as potent as kojic acid, the positive control.

Keywords: alaternin; anthraquinone; glycoside; 6-methoxysorigenin; Rhamnus nakaharai; tyrosinase. 
Figure S1-1: ${ }^{1} \mathrm{H}$ NMR spectrum of Alaternin-8-O-glucopyranoside (1)

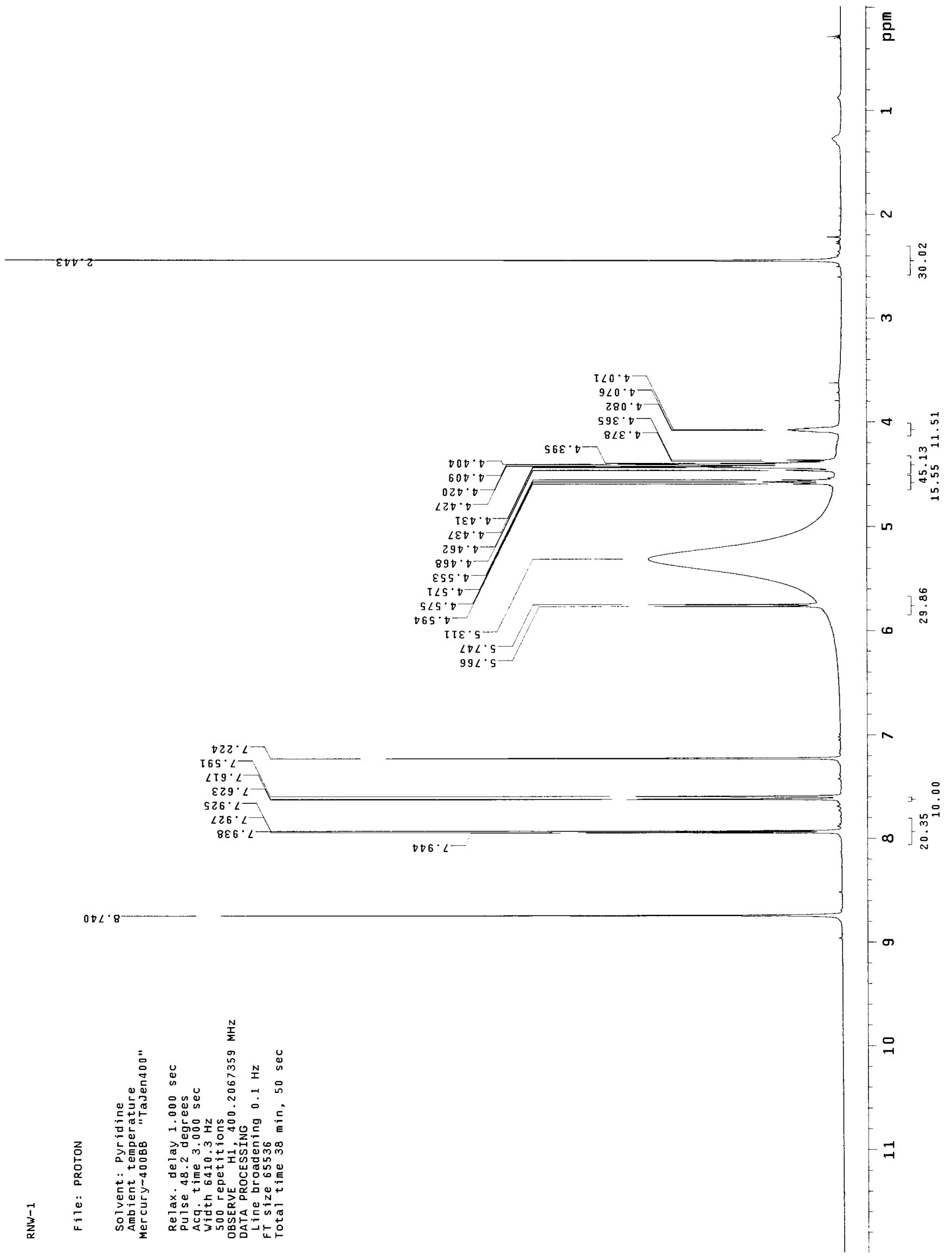


Figure S1-2: ${ }^{13} \mathrm{C}$ NMR spectrum of Alaternin-8-O-glucopyranoside (1)

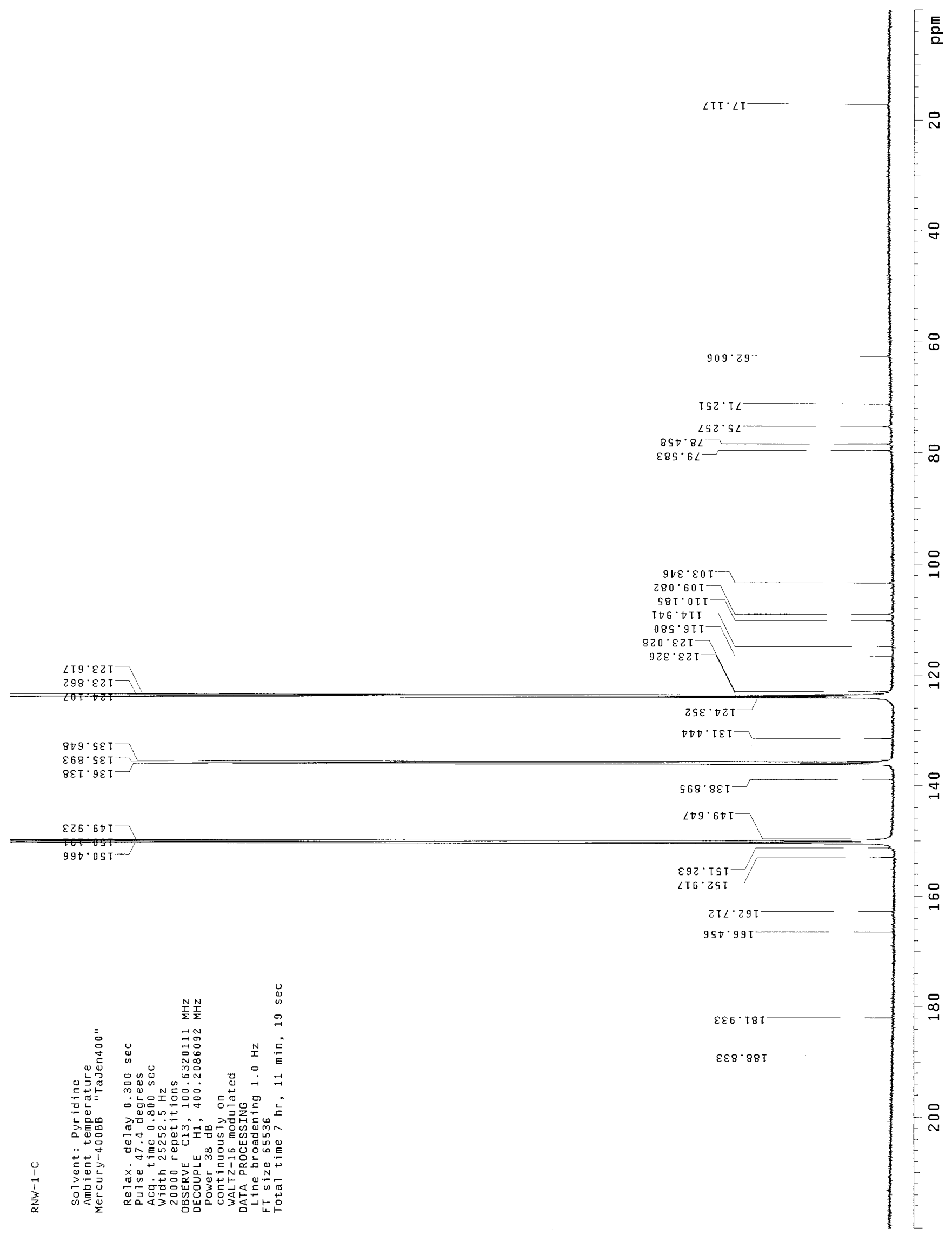


Figure S1-3: HMQC spectrum of Alaternin-8-O-glucopyranoside (1)

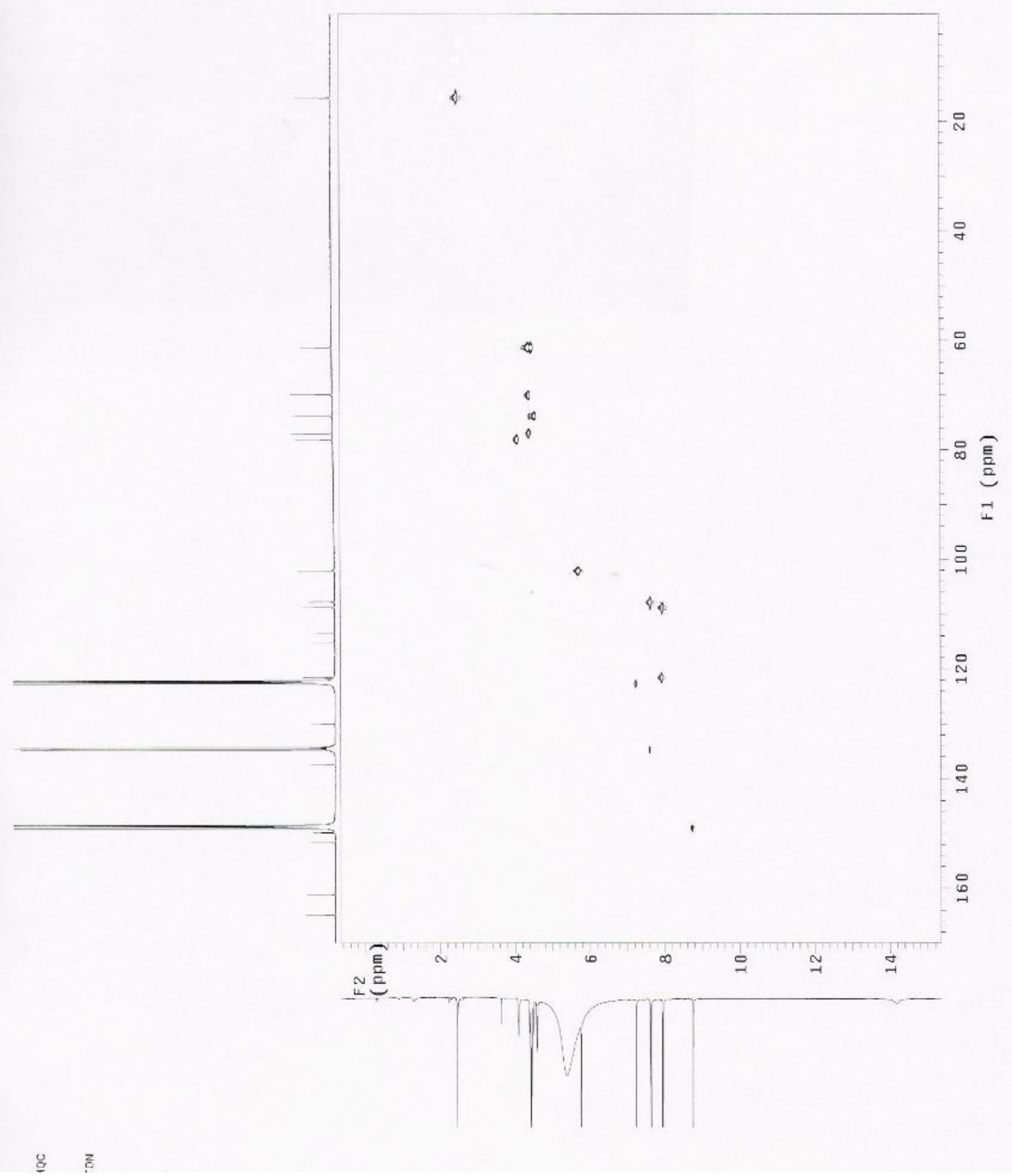


Figure S1-4: HMBC spectrum and key information of Alaternin-8-O-glucopyranoside (1)

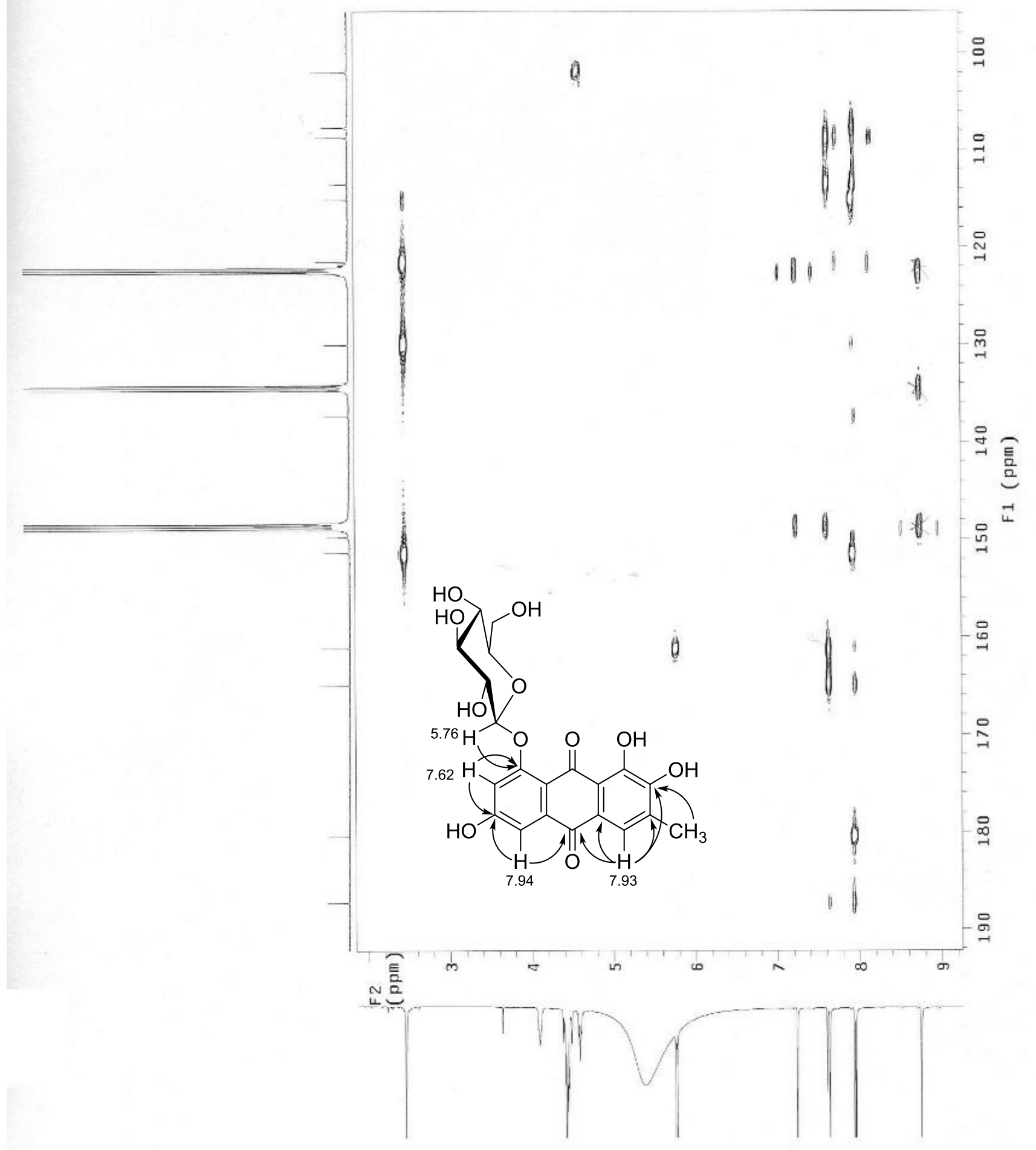


Figure S1-5: FAB-MS spectrum of Alaternin-8- $O$-glucopyranoside (1)

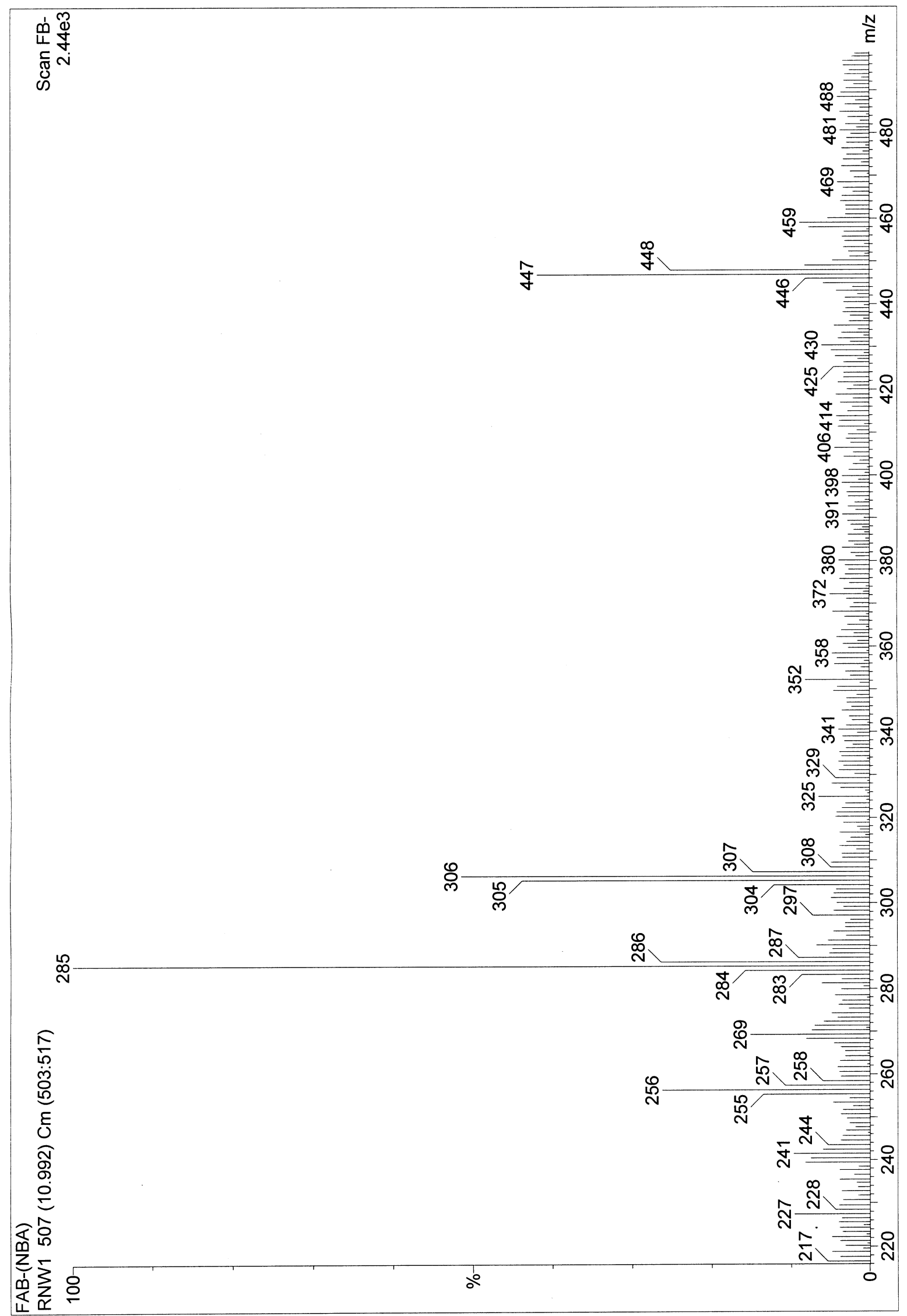


Figure $S$ 2-1: HMQC NMR spectrum of emodin-8-O-glucopyranoside (2)

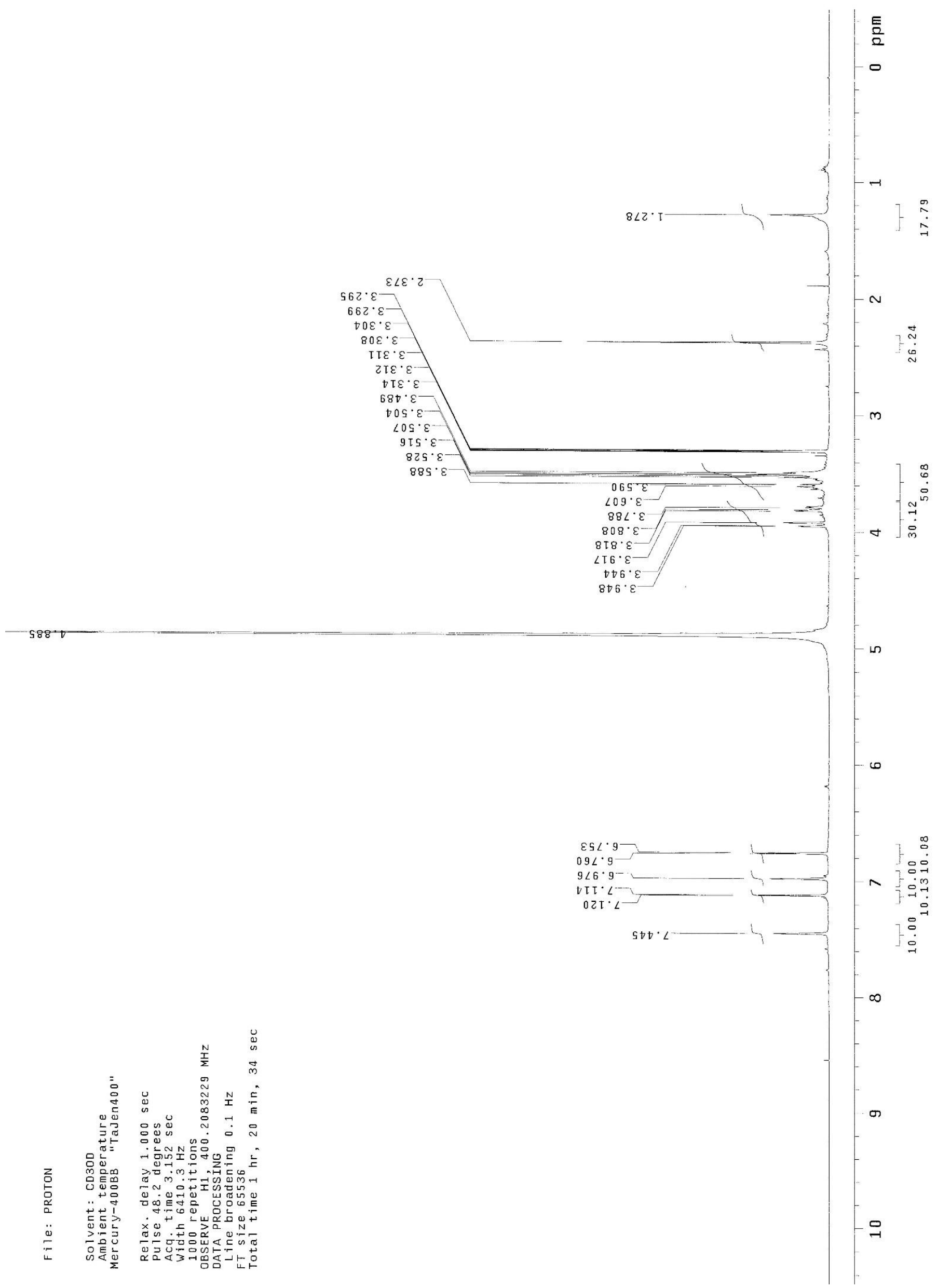


Figure $S 2-2:{ }^{13} \mathrm{C}$ NMR spectrum of emodin-8-O-glucopyranoside (2)

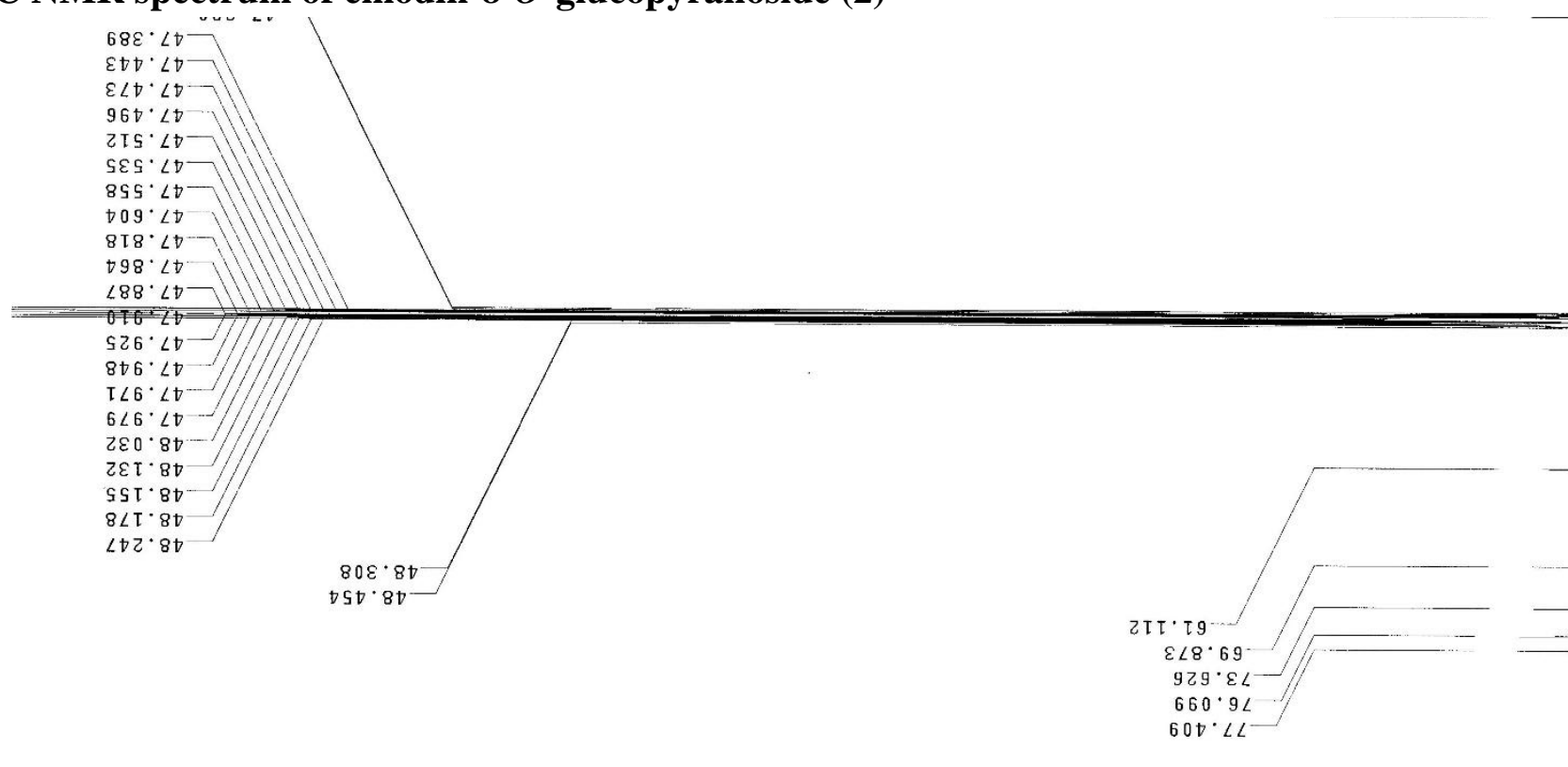

$\varepsilon s 9 \cdot \varepsilon 0 \tau$

$0 \varepsilon 9 \cdot 80 T$

S96. $2 \tau T$ $8 \varepsilon \mathrm{D}^{\circ} \mathrm{st}$ $706.5 T \mathrm{~T}$ $\angle O T \cdot G I T$ $608^{\circ} \varepsilon 2 \mathrm{~T}$ $\angle 6 \tau \cdot \varepsilon \varepsilon \tau$ $829 \cdot 9 \varepsilon \tau-$

Figure $S 2-3$ : DEPT pulse sequence spectrum of emodin-8-O-glucopyranoside (2) 


$$
11
$$

